\title{
Die Medizin auf dem Weg zum Frauenberuf
}

\author{
Nora Willea, Jürg Schlup ${ }^{b}$ \\ ${ }^{a}$ Dr. phil., persönliche wissenschaftliche Mitarbeiterin des Präsidenten; ${ }^{b}$ Dr. med., Präsident der FMH
}

Seit vor gut 150 Jahren die erste Schweizerin an der Universität Zürich ein Medizinstudium abschloss, hat sich in der Ärztedemografie viel getan. Mittlerweile stellen Frauen einen grossen Teil der Ärzteschaft - und voraussichtlich werden sie in naher Zukunft sogar die Mehrheit bilden. Grund genug, diesen Trend genauer anzusehen und viele damit verbundene Themen - Interessensgebiete, Teilzeitarbeit, Karrierechancen und Gender pay gap - etwas zu beleuchten.

Seit Marie Heim-Vögtlin vor etwa 150 Jahren als erste Schweizerin an der Universität Zürich ein Medizinstudium abschloss und 1874 eine Praxis eröffnete [1], haben sich die Geschlechteranteile in der Medizin fundamental gewandelt. Im Jahr 1950 stellten Frauen 12 Prozent der Gesamtärzteschaft - ein Anteil, der bis 1960 gar nicht und auch anschliessend nur langsam wuchs, bis er im Jahr 1980 bei etwa 18 Prozent und 1990 bei 22 Prozent lag. Seither hat sich der Anteil knapp verdoppelt. Die Ärztestatistik des Jahres 2018 zeigt, dass aktuell von 37525 in der Schweiz berufstätigen Ärztinnen und Ärzten 15982 und damit 43 Prozent Frauen sind [2].

\section{Junge Frauen, alte Männer? Demografie der Ärzteschaft}

Ein Blick auf die in den letzten Jahren erteilten eidgenössischen Diplome in Humanmedizin zeigt, dass sich

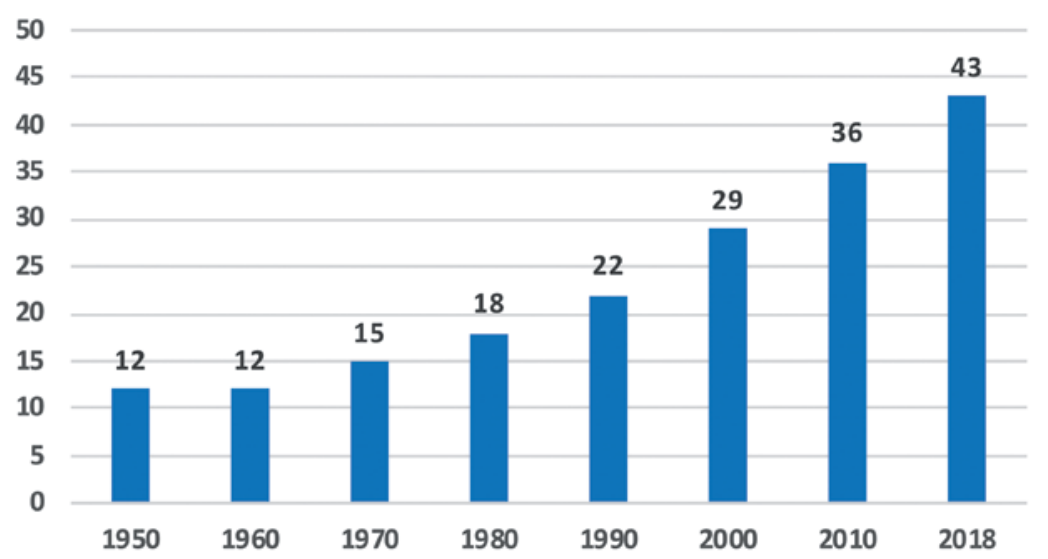

Abbildung 1: Frauenanteil an der berufstätigen Ärzteschaft in Prozent dieser Trend hin zu mehr Frauen in der Medizin fortsetzt: Seit 2005 überwogen jedes Jahr die Frauen unter den Absolventen und Absolventinnen. Und auch bei den anerkannten ausländischen Diplomen überwogen jüngst die Ärztinnen [3]. Demzufolge stellen die Frauen in den jüngeren Generationen der Ärzteschaft bereits die Mehrheit der Berufstätigen, in den älteren Jahrgängen überwiegen noch die Männer (Abb. 2). Sollte sich diese Entwicklung so fortsetzen und nicht durch andere Faktoren relativiert werden (zuwandernde Ärzte, Berufsausstiege), ist absehbar, dass in spätestens 20 Jahren, wenn die heute über 45-Jährigen am Ende ihres Berufslebens stehen, der Arztberuf ein Ärztinnenberuf sein wird.

\section{Frauenfächer - Männerfächer? Veränderungen in den Fachrichtungen}

Der Anteil der Ärztinnen unterscheidet sich jedoch deutlich in den verschiedenen Fachgebieten der Medizin. Die erste Schweizer Ärztin, Marie Heim-Vögtlin, bildete sich als erste Frau in Europa zur Fachärztin für Geburtshilfe und Frauenkrankheiten fort. Betrachtet man die zwischen 2011 und 2018 erteilten Weiterbildungstitel (Tabelle 1), zeigt sich, dass die Gynäkologie und Geburtshilfe mit einem Frauenanteil von $84 \%$, gefolgt von der Kinder- und Jugendmedizin (79\%) und -psychiatrie (77\%) nach wie vor ein Fach ist, in dem sich Ärztinnen häufiger weiterbilden. Auch die grösste ärztliche Fachdisziplin - die Allgemeine Innere Medizin wird aktuell zu 60\%, und damit überproportional häufig, von Frauen gewählt. Im Jahr 2000 waren Frauen unter den Fachärztinnen und Fachärzten für Allgemeinmedizin bzw. Innere Medizin mit 16\% noch unterrepräsentiert. 


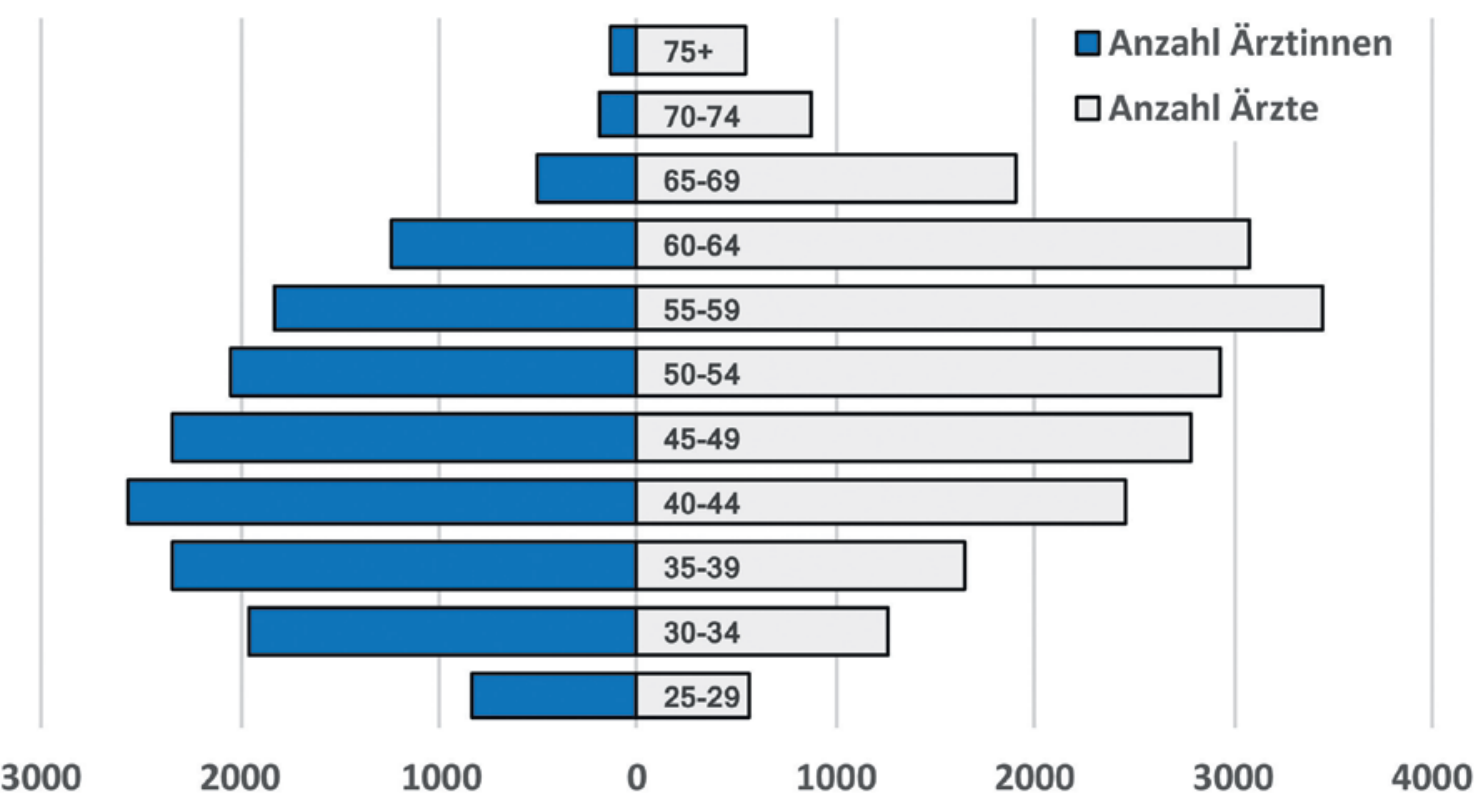

Abbildung 2: Demografie der Ärztinnen und Ärzte nach Altersgruppe (Datenstand 31.12.2018, Aktualisierung: 19.03.2019)

1 Dass sich die Wochenarbeitszeit der Gesamtärzteschaft zwischen 2000 und 2018 stärker reduziert hat als die Wochenarbeitszeit der Frauen bzw. die Wochenarbeitszeit der Männer (Tab. 2), kann nur daraus resultieren, dass die Ärztinnen mit durchschnittlich niedrigerem Pensum einen grösseren Teil der Gesamtärzteschaft stellen.

2 Zu beachten ist zudem, dass die Daten in Tabelle 2 nur eine Reduktion um Halbtage abbilden können: Hätte die Ärzteschaft auch die Dauer ihrer Halbtage im Durchschnitt etwas verkürzt, wäre dies in Tabelle 2 nicht sichtbar.
Was als «Frauenfach» und was als «Männerfach» gilt, unterliegt also einem stetigen Wandel. Dies zeigt sich auch bei mehreren Fächern, die eher als «Männerdomänen» gelten: Während der Anteil der Fachärztinnen im Fach Chirurgie im Jahr 2000 noch bei 5\% lag, betrug er 2018 bereits $23 \%$ - und macht bei den erteilten Weiterbildungstiteln der letzten Jahre sogar $40 \%$ aus (Tab. 1). Auch in Fächern wie der orthopädischen Chirurgie, der Urologie, der Gastroenterologie oder der Neurochirurgie deuten die Frauenanteile bei den Weiterbildungstiteln mit 18 bis 35\% einen Wandel an, wenn man bedenkt, dass Frauen in all diesen Fächern im Jahr 2000 weniger als fünf Prozent der berufstätigen Fachärztinnen und Fachärzte ausmachten.

\section{Arbeitspensen: Wenn weniger als 56 Wochenstunden «Teilzeit» ist}

Gemäss aktuellen Zahlen beträgt die durchschnittliche Arbeitszeit für ein Vollzeitpensum in der Ärzteschaft 55.7 Wochenstunden. Betrachtet man alle Ärzte und Ärztinnen - einschliesslich der Teilzeitarbeitenden - beträgt die durchschnittliche Wochenarbeitszeit 49 Stunden [4]. Vor diesem Hintergrund wird die zunehmende Nachfrage nach Teilzeitarbeit in der Ärzteschaft verständlich: Über die Hälfte der Ärztinnen arbeitet nicht mehr als 70-80\% [5], gemessen am oben genannten Vollzeitpensum also etwa 39 bis 45 Wochenstunden.
Da Ärztinnen häufiger Teilzeit arbeiten als Ärzte, kann sich mit einem höheren Frauenanteil in einer Disziplin auch die für die Patientenversorgung verfügbare ärztliche Arbeitszeit reduzieren. Tatsächlich hat sich die ärztliche Wochenarbeitszeit - gemessen in der Zahl der Halbtage à 4 bis 6 Stunden - zwischen 2000 und 2018 vor allem aufgrund des grösseren Frauenanteils reduziert $^{1}$ - aber: Auch das durchschnittliche Arbeitspensum der Männer ist gesunken. ${ }^{2}$

\section{Berufsausstieg: ein Frauenproblem, ein Arbeitszeitproblem, ein Vereinbarkeits- problem?}

Zur Frage, ob Ärztinnen tatsächlich häufiger aus dem Beruf aussteigen als Ärzte, liegen widersprüchliche empirische Befunde vor [6, 7]. Die Gründe für die Aufgabe der kurativen Tätigkeit unterschieden sich gemäss einer 2016 durchgeführten Befragung zwischen den Geschlechtern jedoch klar: Frauen nennen als häufigsten Grund die mangelnde Vereinbarkeit des Arztberufs mit der Kinderbetreuung (41\%), an zweiter Stelle folgen bei ihnen das hohe Arbeitspensum und die $\mathrm{Ar}$ beitszeiten (40\%). Das Arbeitspensum und die Arbeitszeiten sind unter den aussteigenden Männern die am häufigsten - wenn auch insgesamt seltener (29\%) genannten Gründe. An zweiter Stelle folgen bei den Männern die Arbeitsinhalte (28\%) [8]. Die mangelnde Vereinbarkeit mit der Familie wird hingegen nur von 6\% der Männer als wichtiger Grund angegeben [7]. 
Tabelle 1: Frauenanteil unter den berufstätigen Fachärzten und Fachärztinnen (2000 und 2018) sowie unter den 2011 bis 2018 erteilten Weiterbildungstiteln in den verschiedenen Fachrichtungen

\begin{tabular}{|c|c|c|c|}
\hline & \multicolumn{3}{|c|}{ Frauenanteil in Prozent ( $n=$ Gesamtzahl der Frauen und Männer) } \\
\hline & FachärztInnen 2000 & FachärztInnen 2018 & Weiterbildungstitel 2011-18 \\
\hline Frauenanteil insgesamt & $20 \%\left(18^{\prime} 230\right)$ & $40 \%(32$ '359) & $54 \%\left(12^{\prime} 968\right)$ \\
\hline Medizinische Genetik* & $30 \%(10)$ & $57 \%(28)$ & $90 \%(10)$ \\
\hline Gynäkologie und Geburtshilfe & $28 \%(915)$ & $63 \%(1898)$ & $84 \%(601)$ \\
\hline Kinder- und Jugendmedizin & $36 \%(868)$ & $63 \%(1885)$ & $79 \%(762)$ \\
\hline Kinder- und Jugendpsychiatrie & $51 \%(340)$ & $65 \%(697)$ & $77 \%(205)$ \\
\hline Rechtsmedizin & $17 \%(29)$ & $55 \%(62)$ & $69 \%(45)$ \\
\hline Endokrinologie/Diabetologie & $21 \%(112)$ & $45 \%(222)$ & $68 \%(78)$ \\
\hline Dermatologie und Venerologie & $37 \%(287)$ & $50 \%(584)$ & $67 \%(124)$ \\
\hline Tropen- und Reisemedizin* & $8 \%(50)$ & $19 \%(31)$ & $67 \%(12)$ \\
\hline Pathologie & $34 \%(129)$ & $49 \%(252)$ & $64 \%(91)$ \\
\hline Prävention und Gesundheitswesen & $25 \%(64)$ & $51 \%(70)$ & $63 \%(30)$ \\
\hline Allgemeine Innere Medizin** & $16 \%(6901)$ & $41 \%(8308)$ & $60 \%(3932)$ \\
\hline Psychiatrie und Psychotherapie & $33 \%(1639)$ & $43 \%(3814)$ & $55 \%(1013)$ \\
\hline Hämatologie & $22 \%(113)$ & $39 \%(199)$ & $54 \%(59)$ \\
\hline Infektiologie & $13 \%(85)$ & $39 \%(203)$ & $54 \%(124)$ \\
\hline Med. Onkologie & $21 \%(188)$ & $42 \%(368)$ & $53 \%(193)$ \\
\hline Allergologie/Immunologie & $22 \%(136)$ & $37 \%(164)$ & $53 \%(53)$ \\
\hline Ophthalmologie & $32 \%(544)$ & $42 \%(1048)$ & $53 \%(234)$ \\
\hline Neurologie & $18 \%(262)$ & $34 \%(623)$ & $53 \%(181)$ \\
\hline Anästhesiologie & $34 \%(799)$ & $45 \%(1551)$ & $51 \%(659)$ \\
\hline Praktischer Arzt / Ärztin & - & $47 \%(1492)$ & $51 \%(1283)$ \\
\hline KI. Pharmakol. und Toxikologie & $24 \%(21)$ & $52 \%(44)$ & $50 \%(22)$ \\
\hline Nephrologie & $17 \%(109)$ & $40 \%(229)$ & $49 \%(92)$ \\
\hline Arbeitsmedizin & $13 \%(61)$ & $37 \%(142)$ & $48 \%(46)$ \\
\hline Radio-Onkologie / Strahlentherapie & $34 \%(29)$ & $36 \%(124)$ & $47 \%(51)$ \\
\hline Pharmazeutische Medizin & $29 \%(34)$ & $36 \%(45)$ & $44 \%(27)$ \\
\hline Radiologie & $19 \%(345)$ & $31 \%(901)$ & $43 \%(404)$ \\
\hline ORL & $15 \%(310)$ & $29 \%(511)$ & $42 \%(139)$ \\
\hline Plastische Chirurgie & $20 \%(110)$ & $28 \%(215)$ & $41 \%(66)$ \\
\hline Chirurgie & $5 \%(912)$ & $23 \%(1188)$ & $40 \%(478)$ \\
\hline Intensivmedizin & $17 \%(283)$ & $33 \%(641)$ & $40 \%(343)$ \\
\hline Kinderchirurgie & $21 \%(52)$ & $40 \%(94)$ & $39 \%(33)$ \\
\hline Rheumatologie & $17 \%(372)$ & $29 \%(459)$ & $38 \%(95)$ \\
\hline Neuropathologie* & - & $17 \%(12)$ & $38 \%(8)$ \\
\hline Angiologie & $16 \%(96)$ & $26 \%(193)$ & $37 \%(62)$ \\
\hline Pneumologie & $10 \%(209)$ & $26 \%(329)$ & $36 \%(117)$ \\
\hline Phys. Medizin und Rehabilitation & $17 \%(192)$ & $31 \%(237)$ & $35 \%(51)$ \\
\hline Handchirurgie & $14 \%(114)$ & $24 \%(194)$ & $35 \%(66)$ \\
\hline Gastroenterologie & $4 \%(239)$ & $19 \%(402)$ & $35 \%(119)$ \\
\hline Kardiologie & $8 \%(378)$ & $20 \%(795)$ & $31 \%(306)$ \\
\hline Nuklearmedizin & $10 \%(48)$ & $22 \%(77)$ & $27 \%(37)$ \\
\hline Urologie & $1 \%(152)$ & $15 \%(353)$ & $23 \%(107)$ \\
\hline Orthopädische Chirurgie & $3 \%(542)$ & $10 \%(1170)$ & $22 \%(443)$ \\
\hline Neurochirurgie & $4 \%(71)$ & $14 \%(217)$ & $18 \%(83)$ \\
\hline Gefässchirurgie & - & $12 \%(81)$ & $16 \%(37)$ \\
\hline Thoraxchirurgie & - & $6 \%(35)$ & $11 \%(18)$ \\
\hline Herz- und thorak. Gefässchirurgie* & $6 \%(35)$ & $12 \%(81)$ & 7\%(14) \\
\hline Mund-, Kiefer- + Gesichtschirurgie* & - & $8 \%(91)$ & 7\% (15) \\
\hline
\end{tabular}

a Quelle: FMH-Statistik «Berufstätige Ärzte nach Facharzttitel», FMH - Ärztestatistik 2000

b Quelle: FMH-Statistik "Berufstätige Ärzte nach Hauptfachgebiet und Geschlecht; 2018», FMH - Ärztestatistik 2018

${ }^{c}$ FMH-Auswertung der zwischen 2011 und 2018 erteilten Weiterbildungstitel

* Disziplinen mit Zellen, die ein $n \leq 15$ aufwiesen, wurden optisch zurückgenommen, um die statistische Unsicherheit aufgrund sehr kleiner

Fallzahlen zu verdeutlichen.

** Für die Angaben des Jahres 2000 wurden die Fächer Allgemeinmedizin und Innere Medizin zusammengefasst. 
Tabelle 2: Durchschnittliches Pensum in Halbtagen pro Woche (ein Halbtag $=4$ bis $6 \mathrm{~h}$ ) nach Sektor und Geschlecht, 2008 \& 2018.

\begin{tabular}{lllcccc}
\hline & \multicolumn{2}{c}{ Frauen } & \multicolumn{2}{c}{ Männer } & \multicolumn{2}{c}{ Alle } \\
& $\mathbf{2 0 0 8}[\mathbf{1 1}]$ & $\mathbf{2 0 1 8}[\mathbf{1 2}]$ & $\mathbf{2 0 0 8}[\mathbf{1 1}]$ & $\mathbf{2 0 1 8}[\mathbf{1 2}]$ & 2008 [11] & 2018 [12] \\
\hline Ambulanter Sektor & 7,1 & 6,9 & 9,1 & 8,8 & 8,7 & 8,1 \\
\hline Stationärer Sektor & 9,0 & 8,8 & 10,2 & 10,1 & 9,9 & 9,5 \\
\hline
\end{tabular}

Quellen: [11] Kraft E, FMH-Ärztestatistik 2008. Neue Zahlen - neuer Inhalt - neues Layout. SAEZ 2009;90: 12; [12] Hostettler S, Kraft E. Wenig Frauen in Kaderpositionen. SAEZ 2019;100(12):411-416

Tabelle 3: Frauenanteil (\%) in den verschiedenen Altersgruppen nach Hierarchieebene ( $\mathrm{n}=$ Gesamtzahl der Frauen und Männer).

\begin{tabular}{|c|c|c|c|c|c|}
\hline & Insgesamt & Assistenzärztinnen & Oberärztinnen & Leitende Ärztinnen & Chefärztinnen \\
\hline $25-29$ & $60 \%(1384)$ & $60 \%(1379)$ & $0 \%(2)$ & $0 \%(0)$ & $0 \%(0)$ \\
\hline $30-34$ & $61 \%(2964)$ & $61 \%(2773)$ & $56 \%(162)$ & $0 \%(4)$ & $50 \%(2)$ \\
\hline 40-44 & $49 \%(2834)$ & $59 \%(1428)$ & $45 \%(801)$ & $27 \%(318)$ & $16 \%(117)$ \\
\hline 45-49 & $41 \%(2236)$ & $55 \%(716)$ & $47 \%(593)$ & $23 \%(486)$ & $15 \%(211)$ \\
\hline 60 plus & $22 \%(1675)$ & $39 \%(228)$ & $38 \%(266)$ & $18 \%(404)$ & $9 \%(463)$ \\
\hline Alle & $47 \%(17609)$ & $59 \%(9295)$ & $48 \%(3294)$ & $25 \%(2151)$ & $12 \%(1555)$ \\
\hline
\end{tabular}

\section{Gibt es eine gläserne Decke? Frauenanteil in verschiedenen Karrierestufen}

Dass sich auch in der Ärzteschaft «Wenig Frauen in $\mathrm{Ka}$ derpositionen" finden und der Frauenanteil mit steigender Hierarchiestufe abnimmt, ist bekannt [12]. Liegt dies nur daran, dass Ärztinnen vor allem in den jüngeren Altersgruppen vertreten sind - und darum (noch) nicht so häufig in Kaderpositionen aufgerückt sind? Die altersstratifizierte Darstellung der Spitalärzte und -ärztinnen nach Hierarchiestufen zeigt, dass diese Annahme nicht zutrifft: In jeder Altersgruppe sind Frauen seltener in den höheren Hierarchiestufen vertreten als aufgrund ihres Anteils in der jeweiligen Altersgruppe zu erwarten wäre. So ist der Frauenanteil in Chefarztfunktionen in der Regel nicht einmal halb so gross wie der Frauenanteil in der jeweiligen Altersgruppe.

\section{Gender pay gap - auch in der Ärzteschaft?}

Als das Bundesamt für Gesundheit im November 2018 mithilfe einer Studie grosse mediale Aufmerksamkeit für die Ärzteeinkommen generierte, behauptete es unter anderem auch "systematische Unterschiede» zwischen den Geschlechtern: «Die Einkommen der Ärzte liegen mit +29 Prozent substanziell über denjenigen der Ärztinnen» - obwohl «Berufserfahrung, Fachgebiet und Tätigkeitssektor berücksichtigt» seien [9]. Sind Ärztinnen also von Lohndiskriminierung betroffen?
Die betreffende Studie des BAG lässt eine solche Aussage kaum zu, zeigt sich doch bei genauem Nachlesen, dass in der zugrundeliegenden multivariaten Analyse «Einflussmerkmale wie z.B. die ausgeübte Funktion (Oberarzt, Leitender Arzt, Chefarzt usw.) fehlen" (10, S. 41). Die Einkommensdifferenz dürfte also auch der Tatsache geschuldet sein, dass Frauen in den hohen Hierarchiestufen deutlich untervertreten sind (siehe Tabelle 3).

Und noch ein weiterer methodischer Makel gibt Anlass zu Zweifeln - nicht nur an den "standardisierten" Einkommen - sondern auch an den behaupteten Geschlechterunterschieden: Da die Wochenarbeitszeit in Halbtagen erfasst wurde, diese aber als Zeitraum von 4 bis 6 Stunden definiert sind, bleiben Arbeitszeitdifferenzen von bis zu 20 Wochenstunden unsichtbar. Hier läge eine erhebliche Fehlerquelle sollten Ärztinnen durchschnittlich nicht nur weniger Halbtage (siehe Tabelle 2) sondern durchschnittlich auch eher kürzere Halbtage arbeiten als ihre männlichen Kollegen. ${ }^{3}$

«Standardisierungen» auf Basis dieser Daten zum Arbeitspensum erlauben folglich keine validen Einkommensvergleiche. Hierfür bräuchte es eine Kenntnis der Stundenlöhne - und weiterer Faktoren wie u.a. Berufserfahrung und Funktion. Da solche Daten nicht vorliegen, kann ein Gender pay gap in der Schweizer Ärzteschaft bislang weder festgestellt noch ausgeschlossen werden. 


\section{Und wann kommen Ärztinnen auch in der Standespolitik an?}

Obwohl Frauen mittlerweile mit 43\% in der berufstätigen Ärzteschaft stark vertreten sind, spiegelt sich dies bislang in standespolitischen Gremien wenig wider. Im Zentralvorstand der FMH ist nur eines der sieben Mitglieder eine Frau (14\%), in der Delegiertenversammlung stellen Frauen 9 von 33 Delegierten (27\%), in der letzten Ärztekammer von Oktober 2019 waren 25 Prozent der Stimmberechtigten Frauen. Auch in den Vorständen der grössten Fachgesellschaften sind Frauen eher untervertreten - teilweise deutlich. Ein Grund dafür könnte sein, dass die Standespolitik überwiegend von einer Ärztegeneration geprägt ist, deren Frauenanteil noch geringer war. Es könnte aber auch die für Frauen bedeutsamere Vereinbarkeitsproblematik einem standespolitischem Engagement im Wege stehen.

\section{Fazit: Ein Berufsstand im Wandel}

Die Zahl der Medizinstudentinnen ist bereits seit vielen Jahren grösser als die der Medizinstudenten und der steigende Anteil an Ärztinnen in sämtlichen ärztlichen Fachdisziplinen fordert alte Denkmuster und Organisationsstrukturen heraus. Besonders die Diskussionen um Arbeitszeiten und die Möglichkeiten und Grenzen von Teilzeitarbeit werden durch diesen demografischen Wandel des Berufsstands befördert. Dies kommt nicht nur jungen Ärztinnen entgegen. Mit dem Wandel der Rollenmodelle dürften auch junge Ärzte heute häufiger den Wunsch verspüren, im Familienalltag präsenter zu sein und seltener eine Lebenspartnerin im Hintergrund haben, die alle Belange ausserhalb des Berufs für sie übernimmt. Für eine Sicherstellung der ärztlichen Versorgung in der
Schweiz wird es neben ausreichend Studienplätzen darum auch Anstrengungen brauchen, den ärztlichen Nachwuchs mit zeitgemässen Arbeitszeitmodellen im Beruf zu halten - und dies in allen zu besetzenden Fachrichtungen. Hierbei sollten auch die Karrierechancen Thema sein. Das Geschlecht darf kein Karrierehindernis sein, genauso wenig wie Familienpausen oder Teilzeitarbeit dem beruflichen Aufstieg im Wege stehen sollten. Für eine starke und in ihrer Basis gut verankerte Standesorganisation ist zu hoffen, dass der Frauenanteil zukünftig auch in der Standespolitik zunehmen wird - und sich vermehrt auch Ärztinnen in der Gestaltung dieses Wandels engagieren.

\section{Referenzen}

1 Naef, J. Die erste Schweizer Ärztin - Dr. med. Marie Heim-Vögtlin. SAEZ 2016;97(9):315-317.

2 Die Ärztestatistiken der jeweiligen Jahrgänge können auf den Webseite der FMH eingesehen werden.

3 Bundesamt für Gesundheit BAG. Direktionsbereich Gesundheitspolitik (2018). Ärztinnen und Ärzte 2018

4 Golder L, Jans, C, Tschöpe S, Venetz A, Herzog N (2018). Verändertes Arbeitsumfeld und Einstellung zu neuen Finanzierungsmodellen. Auswirkungen Leistungsorientierung im Gesundheitswesen erkennbar. Schlussbericht gfs.bern

5 FMH Ärztestatistik Poster 2018

6 One in seven Swiss physicians has left patient care - results from a national cohort study from 1980-2009. Swiss Med Wkly, 2019;149:w20116

7 Kraft E, Loretan L, van der Heiden N. Jeder zehnte Arzt steigt aus. SAEZ 2016;97(34):1132-1135. https://doi.org/10.4414/saez.2016.04953.

8 Bolliger C, Golder L, Jans C. Der Ausstieg aus der kurativen Tätigkeit. Schlussbericht. gfs.bern; 31. Mai 2016.

9 Medienmitteilung des BAG, 29.10.2018. Einkommen von Ärztinnen und Ärzten in der Schweiz: Neue Studie bringt Transparenz.

10 Künzi K, Morger M. (2018). Einkommen, OKP-Leistungen und Beschäftigungssituation der Ärzteschaft 2009-2014. Analyse verknüpfter Datensätze: Medizinalberuferegister BAG, AHV-Daten der Zentralen Ausgleichsstelle, Krankenversicherungsdaten SASIS. Schlussbericht. Im Auftrag des Bundesamts für Gesundheit BAG. Direktionsbereich Gesundheitspolitik.

11 Kraft E, FMH-Ärztestatistik 2008. Neue Zahlen - neuer Inhalt neues Layout. SAEZ 2009;90: 12 .

12 Hostettler S, Kraft E. Wenig Frauen in Kaderpositionen. SAEZ 2019;100(12):411-416

\section{Coronavirus}

Aktuelle Informationen rund um COVID-19 erhalten Sie unter: 